\title{
Optimal base point selection method based on convex hull construction technology
}

\author{
Jian Dong ${ }^{1}$, Zhiqiang Zhang ${ }^{1}$, Lulu Tang ${ }^{1}$, Rencan Peng ${ }^{1}$, Hongchao $\mathrm{Ji}^{1,2}$ \\ ${ }^{1}$ Department of Military Oceanography and Hydrography \& Cartography, Dalian Naval Academy, Dalian 116018, China \\ ${ }^{2}$ Chart Information Centre, Tianjin 300450, China
}

\begin{abstract}
The primary purpose of maritime delimitation is to ensure the maximum internal waters area obtained. In order to grantee the maximum internal waters area obtained with the selected base point, the idea and method of optimal selection of the territorial sea base points with the convex hull (minimum convex hull) construction technology is proposed. The ideal base points are selected by constructing convex hull for all alternative base points, which makes it possible to realize the automatic selection of base points under the principle of the maximum internal waters area.
\end{abstract}

\section{Introduction}

According to the "United Nations Convention on the Law of the Sea", coastal states may decide to adopt a normal baseline method, a straight baseline method or a hybrid baseline method combining the two according to actual conditions ${ }^{[1]}$. When adopting the straight baseline method, the different selection schemes of base points in the territorial sea, which means that the number of territorial sea base points selected and their spatial point distribution are different, have an important impact on the result of demarcation. Because once the position of the base point is determined, it means that the baseline connecting the base point and the starting line of the internal waters, territorial sea, contiguous zone and exclusive economic zone based on the baseline will be determined accordingly ${ }^{[2]}$. However, in the traditional manual base point selection work, due to the limitation of technical constraints, it is difficult to provide a base point selection and evaluation model that meets these regulations. Therefore, it is impossible to ensure that the selected base point meets the requirements of the United Nations Convention on the Law of the Sea, so as to achieve the purpose of obtaining the maximum internal waters area ${ }^{[3]}$. In this paper, based on the analysis of the concept and generation technology of convex hull, the minimum convex hull construction of the alternative base point is used to generate the convex hull boundary point conforming to the characteristics of maximum geometric area, so as to realize the optimal selection of the territorial sea base point under the principle of maximum internal waters area.

\section{Related concepts of convex hull}

(1) Simple polygon: Polygons whose edges do not intersect except for vertices (non-adjacent sides do not intersect) are called simple polygons ${ }^{[4-5]}$. The polygons involved in this article are all this type of polygons. Simple polygons can be divided into convex polygons and arbitrary polygons;

(2) Convex polygons: Polygons with all interior angles less than $\pi$ are called convex polygons ${ }^{[4 \sim 5]}$. All vertices of a convex polygon are convex points.

(3) Convex hull (also known as minimum convex hull or minimum convex set ) : Let $S$ be the point set in the plane ( $E^{2}$ ), and use $C H(S)$ to represent the convex hull of the point set $S, B C H(S)$ represents the convex hull boundary of $\mathrm{CH}(S)$.

(4) Convex hull vertex: If the vertex sequence $P_{1}, P_{2}, P_{3}, \ldots \ldots, P_{n}$ of the convex hull polygon $P$ are arranged in a counterclockwise direction, the convex hull vertex $P_{\mathrm{i}+1}$ is on the left side of $\overrightarrow{P_{i-1} P_{i}}$.

Related definitions of convex shells are as follows [3 5$]$ :

Definition 1 Let $S$ be a non-empty set of points on the plane, $P_{1}, P_{2}$ be any two points in $S$, and $S$ be a convex set if point $p=t p_{1}+(1-t) p_{2}$ is a member of $S$, where $0 \leq t \leq 1$. That is, if any two points of connection in $S$ are all in $S$, then $S$ is convex.

Definition 2 A convex shell $C H(S)$ of a plane point set $S$ is the smallest convex hull containing $S$. 
Definition 3 The boundary $B C H(S)$ of the convex hull of the plane point set $S$ is a convex polygon whose vertices are points in $S$.

$B C H(S)$ is the smallest convex polygon $P$ surrounding $S$, that is, there is no polygon $P^{\prime}$, so that $P \supset P^{\prime} \supset S$ is established.

$B C H(S)$ is a closed convex polygon $P$ with the smallest area, or a closed convex polygon $P$ with the smallest perimeter.

The convex hull or convex hull boundary of the plane point set $S$ is a convex polygon; on the contrary, a convex polygon must be the convex hull or convex hull boundary of a plane point set.

\section{Convex hull generation algorithm}

There are many kinds of convex hull generation algorithms for discrete point sets. Let $S$ be the point set of points on the plane $n$, then it takes at least $O(n \log n)$ time to calculate the convex hull. Because the time complexity of the method proposed by Graham is $O(n \log n)$, the method proposed by Graham is the best algorithm for solving the convex hull of a plane point set ${ }^{[3]}[6]$.

The basic basis of Graham Algorithm for generating the convex hull of a point set is: all vertices of a convex polygon must be on the same side of any side of the polygon. As shown in Figure 1, assuming a discrete point set $S=\left\{P_{1}, P_{2}, \ldots \ldots, P_{n}\right\}$, the specific steps of Graham Algorithm to generate the convex hull of the point set are as follows ${ }^{[7 \sim 9]}$ :

(1) Find the point $P_{1}$ with the Minimum $y$ coordinate: $P_{1}=\min \left(y_{i}\right)$.

(2) $P_{1}$ is connected with other points $P_{i}(i \neq 1)$ to form a line segment $P_{1} P_{i}$, and the angle $\theta_{i}(1<i \leq n)$ between these line segments and the horizontal line is calculated.

(3) Sort by the angle size to get a sequence $P_{1}, P_{2}, \ldots \ldots, P_{n}$, connect these points in turn, and get a polygon (Figure 1(b)). Then point $P_{1}$ is the starting point of the boundary of the convex hull, and $P_{2}, P_{n}$ must also be the vertex of the convex hull, and $P_{n+1}=p_{1}$.

(4) Determine whether the points $P_{3}, P_{4}, \ldots \ldots, P_{n-1}$ are the vertices of the convex hull, and delete the points in $P_{3}, P_{4}, \ldots \ldots, P_{n-1}$ that are not the vertices of the convex hull.

The specific criterion is: for $P_{i}(i=3, i<n)$,

(1) If $P_{i-2}$ and $P_{i+1}$ are on the same side of $P_{i-1} P_{\mathrm{i}}$, namely

$$
\left(\overrightarrow{P_{i-2} P_{i-1}} \times \overrightarrow{P_{i-1} P}\right) \cdot\left(\overrightarrow{P_{i-1} P_{i}} \times \overrightarrow{P_{i} P_{i+1}}\right) \geq 0,
$$

$P_{i}$ is the vertex of the convex hull. Then, keep that point, and continue to judge the next point (as shown in Figure 1€);

(2)If $P_{i-2}$ and $P_{i+1}$ are on both sides of $P_{i-1} P_{\mathrm{i}}$, namely

$$
\left(\overrightarrow{P_{i-2} P_{i-1}} \times \overrightarrow{P_{i-1} P_{i}}\right) \cdot\left(\overrightarrow{P_{i-1} P_{i}} \times \overrightarrow{P_{i} P_{i+1}}\right)<0,
$$

$P_{i}$ the vertex of the convex hull. Then, delete that point, and reduce the number of subsequent vertices by 1 (as shown in Figure 1(d), €).

(5) Output the vertices of the convex hull in sequence (Figure 1(f)).

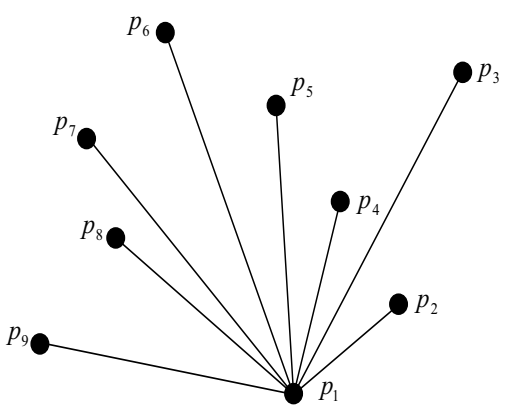

(a)

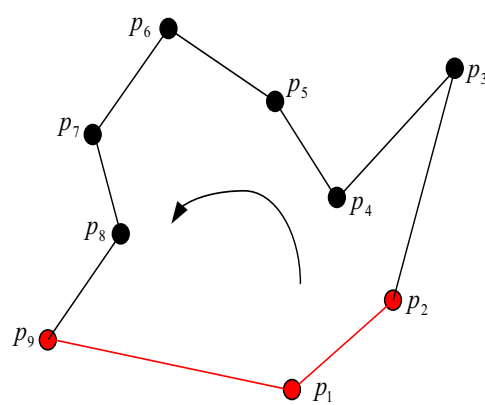

(b)

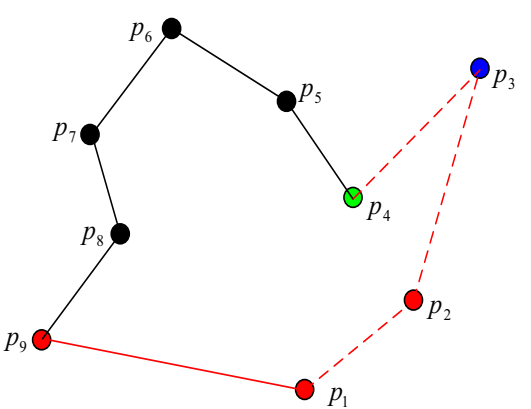

(c) 


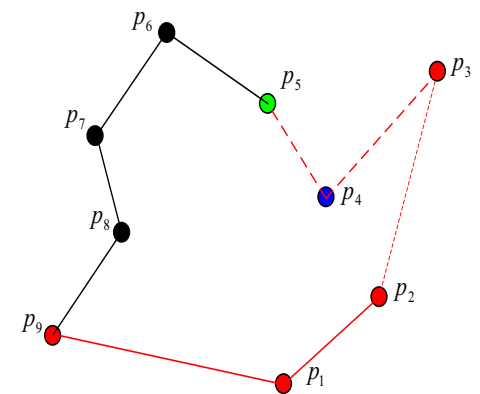

(d)

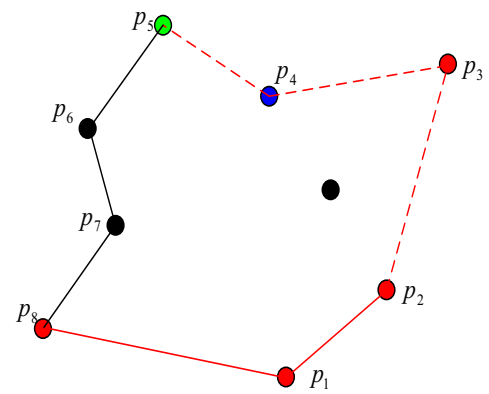

$€$

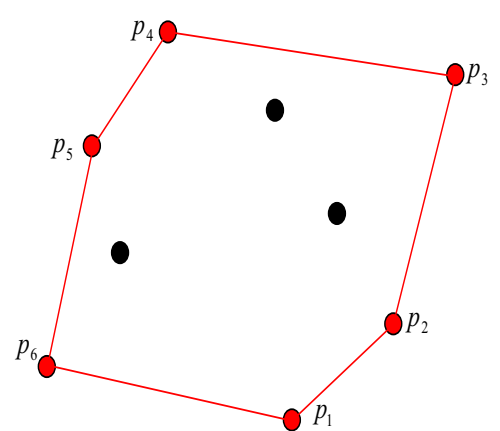

(f)

Figure 1. Schematic diagram of the convex hull of the point set generated by the Graham algorithm.

From the description above, we can know that, for a given set of discrete points, the corresponding algorithm can be used to obtain the only minimum convex polygon corresponding to it. That is, for the discrete point set $P$,its smallest convex hull $B C H(S)$ is such a polygon. If there is another convex polygon $P_{0}$ containing, then $B C H(S) \subseteq P_{0}$, so $B C H(S)$ is the smallest convex polygon containing the discrete point set $P$.

\section{Determine ideal base point based on convex hull construction technology}

On the basis of determining the alternative base point, the convex hull construction technique is used to generate the minimum convex hull of the alternative base point set. For all alternative base points given, the inner waters area enclosed by the smallest convex is the largest. The selected base point of the smallest convex hull polygon can be regarded as the ideal base point, so as to determine the territorial sea base point that satisfies the principle of maximum internal waters area. The specific steps for determining the ideal base point based on the

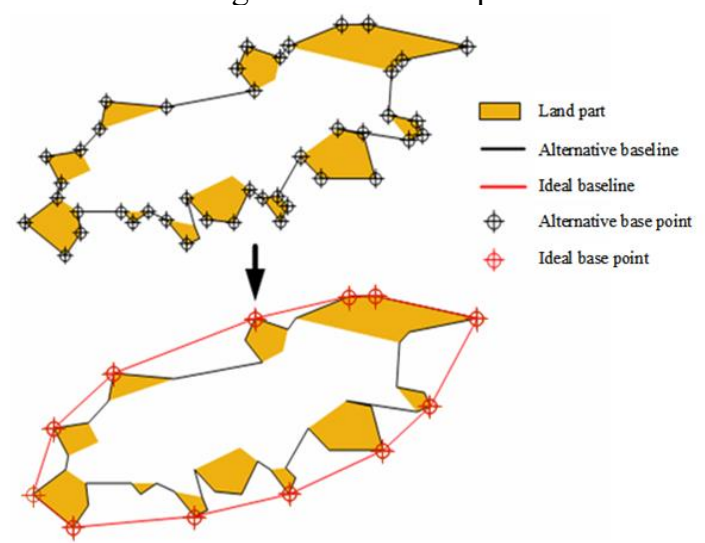

(a) Selection of ideal base points of archipelagos convex hull construction technology are as follows (as shown in Figure 2):

(1) First, extract all the points that are eligible to be the base points of the alternative territorial waters (such as the points that constitute the continental low-water line, the low-water line of the island, and the edge of the low-tide elevation) to form a discrete point set, which is the alternative base point set;

(2) Convex hull generation algorithm is adopted to automatically generate convex hull polygons of alternative base point set:

(1) If it is an archipelago alternative base point, that is, the alternative base point can form a closed polygon to express the outer edge of the archipelago, so the convex hull can be directly constructed, as shown in Figure 2(a);

(2) If it is a continental alternative base point, that is, the alternative base point cannot form the outer edge of the closed polygon expression area, you can first add a sealing line (point), and then construct a convex hull, as shown in Figure 2(b).

(3) Extract all the vertices of the convex hull polygon (remove the sealing point), which is the ideal base point of territorial sea.

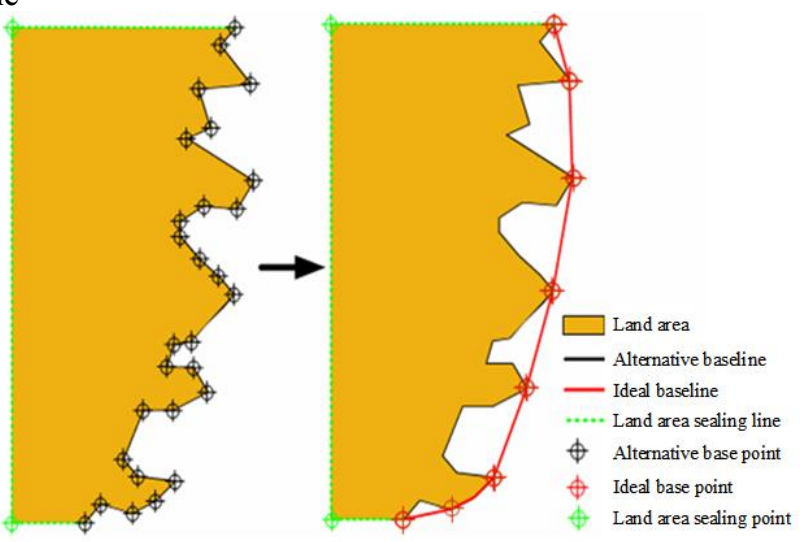

(b) Selection of the ideal base point of the mainland

Figure 2. Determines the ideal base point based on the convex hull construction technology.

\section{Test and analysis}

In order to verify the effectiveness of the algorithm, a section of coastal low tide line is selected to carry out the ideal base point determination and optimization test. The test data is shown in Figure 3(a). As shown in Figure $3(\mathrm{~b})$, first add land sealing points to the alternative base point, and then construct the convex hull to obtain the convex hull vertex. The apex of the convex hull (except 
the sealing point) is the ideal base point. The territorial sea base point selected in this way can ensure the largest internal waters area, as shown in Figure 3(c). Table 1 shows the length of each ideal baseline after the base point is selected based on the convex hull construction technology.

It can be seen from Figure 3 that the convex hull (smallest convex hull) generated by the alternative base points ensures the maximum internal waters area. Therefore, the method of selecting territorial sea base points based on the convex hull construction technology can be regarded as an ideal mathematical model for base point selection. It is conducive to the scientific selection of base points and the improvement of the accuracy of territorial sea base point selection, which is expected to free the base point selection from the long-term completely empirical and manual selection method, which provides the possibility for the scientific selection of territorial sea base point.

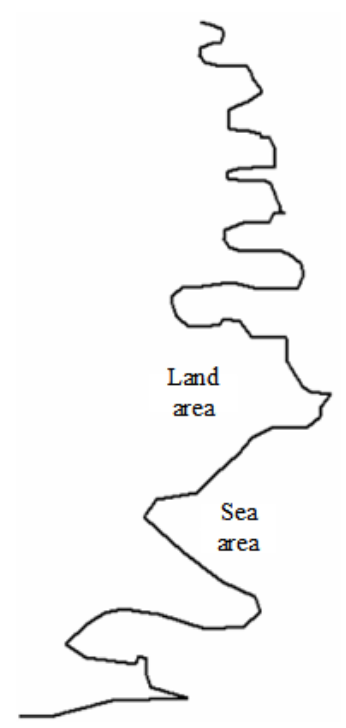

(a)Alternative base point and baseline

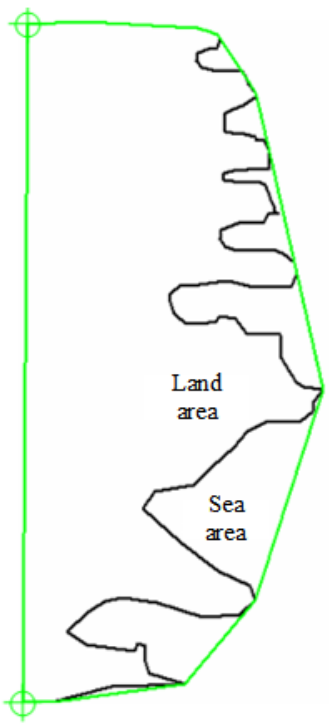

(b) Add land sealing point and convex hull constructionand baseline

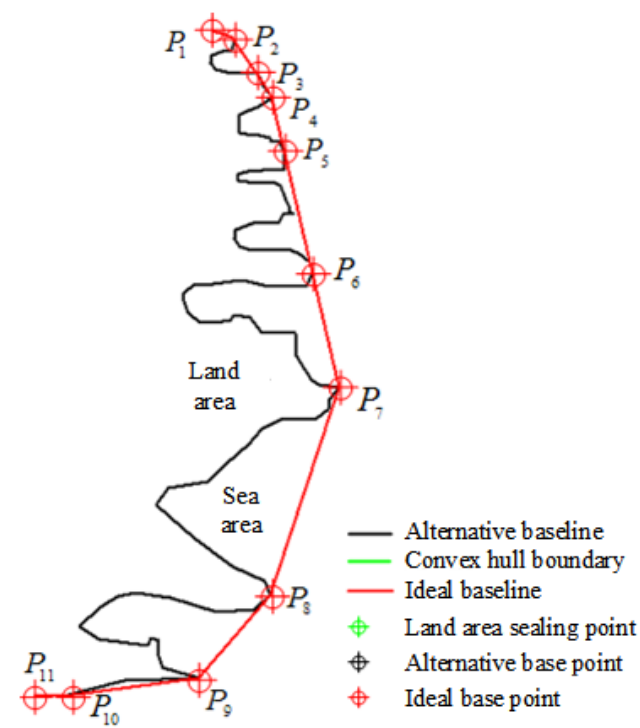

(c) Determination of ideal base point and baseline

Figure 3. Arbitrary polygon connected by alternative base points and ideal base points determined based on convex hull construction technology.

Table 1. Line segments and their lengths that make up the smallest convex hull.

\begin{tabular}{ccccccccccc}
\hline Line segment & $P_{1} P_{2}$ & $P_{2} P_{3}$ & $P_{3} P_{4}$ & $P_{4} P_{5}$ & $P_{5} P_{6}$ & $P_{6} P_{7}$ & $P_{7} P_{8}$ & $P_{8} P_{9}$ & $P_{9} P_{10}$ & $P_{10} P_{11}$ \\
\hline Length (/n mile) & 6.21 & 10.53 & 7.34 & 18.33 & 42.60 & 45.71 & 103.52 & 38.61 & 37.22 & 8.05 \\
\hline
\end{tabular}

The ideal base point given by the convex hull point position can ensure the largest internal waters area, but the base point must also be selected in accordance with the relevant provisions of the United Nations Convention on the Law of the Sea. It is generally considered that the length of the baseline cannot be too long to ensure that the baseline does not deviate significantly from the shoreline. It can be seen from Table 1 that after using the convex hull structure technology to determine the ideal base point, some ideal baseline lengths are too long (such as $P_{5} P_{6} 、 P_{6} P_{7} 、 P_{7} P_{8}$ in Table 1). Therefore, the ideal base point determined based on the convex hull structure technology cannot be used directly. The formal base point of the territorial sea needs to be further optimized for the ideal base line with too long length (such as $P_{5} P_{6} 、 P_{6} P_{7} 、 P_{7} P_{8}$ in Table 1) to ensure that the final base point meets the relevant provisions of the United Nations Convention on the Law of the Sea. The basic idea is to insert appropriate alternative base points between ideal base points whose length exceeds the limit to achieve the purpose of shortening the baseline length, so that the base point can ensure the largest internal waters area under the premise of meeting the requirements of the United Nations Convention on the Law of the Sea.

\section{Conclusion}

The method of selecting ideal territorial water base points introduced in this article is to obtain the ideal territorial water base points by constructing polygon convex hulls based on the determination of candidate base points by means of human-computer interaction and then forming all candidate base points into polygons. Although many tools of GIS can be used to improve the efficiency of this operation and reduce labor intensity to some extent, the traces of manual operation are still retained, and the 
degree of automation and efficiency need to be further improved. In addition, due to the restrictions on the length of the baseline in the United Nations Convention on the Law of the Sea, the next step is to further study the optimization of the base point of the territorial sea under the condition of the limit of the baseline length.

\section{Acknowledgments}

Foundation support: The National Key R\&D Program of China, No. 2017YFC1405505; The National Natural Science Foundation of China (42071439, 41871369 and 41901320).

\section{References}

1. United Nations convention on the law of the sea. Beijing: Maritime Press, 1992.

2. Haiwen Zhang, Haiqing Li. (2005) Interpretation of "United Nations convention on the law of the sea". Beijing: Maritime Press.

3. Rencan Peng, Jiayao Wang, Zhen Tian, et al. (2005) A Research for Selecting Baseline Point of the Territorial Sea Based on Technique of the Convex Hull Construction. J. Acta Geodaetica et Cartographica Sinica, 34(1): 53-57.

4. Rencan Peng. (2003) Research and Practice of Maritime Boundary Delimitation GIS Technology. Information Engineer University, Zhengzhou.

5. Deqing Liang. (2001) Research on maritime boundary delimitation technology. Dalian Naval Academy, Dalian.

6. Rencan Peng, Jiayao Wang. (2002) Study on the Construction Technology of The Earth's Ellipsoidal Buffer Zone. J. Acta Geodaetica et Cartographica Sinica, 31(3): 270-273.

7. Rencan Peng, Jian Dong, Yi Chen, et al. (2009) Research on Maritime Delimitation Model of High Accuracy Equiratio Method. J. Hydrographic Surveying and Charting, 29(4): 14-18.

8. Jian Dong. (2009) Research and Realization of the High-precision Distance Isometric Law. Dalian Naval Academy, Dalian.

9. Jianhui Zhang, Jiye Jin. (2013) Marine Delimitation Method Based on Earth Ellipsoid Model. J. Science of Surveying and Mapping, 38(3): 16-17. 